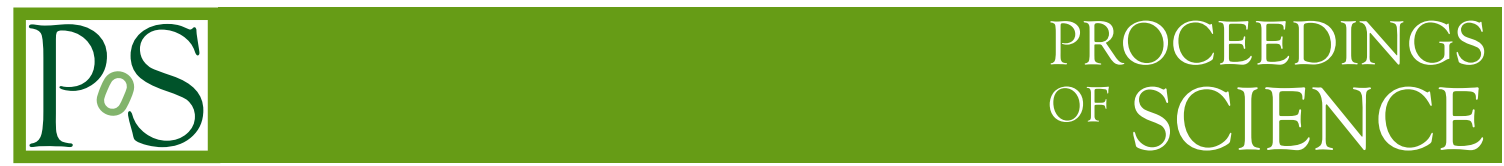

\title{
Ultra fast Nuclear Emulsion Readout System HTS
}

\section{Masahiro Yoshimoto*, T. Nakano, R. Komatani}

Graduate School of Science, Nagoya University, Nagoya 464-8602, Japan

E-mail: yoshimoto@flab.phys.nagoya-u.ac.jp

\begin{abstract}
Automatic nuclear emulsion readout system has been developed at Nagoya University since 1970s. To perform new experiments with much larger area of nuclear emulsion, a new system HTS with almost 100 times faster speed were developed in 2015. We will report the design, the performance and detectability of the shorter tracks.
\end{abstract}

The 3rd International Symposium on "Quest for the Origin of Particles and the Universe" 5-7 January 2017

Nagoya University, Japan

* Speaker. 


\section{Introduction}

Nuclear emulsion have developed ingenious research on cosmic ray, high energy physics owing to its high spatial resolution. In Nagoya University automatic nuclear emulsion readout system has been developed and improved since the 1970s. In order to realize even larger scale experiment, we have developed a new generation readout system HTS. The HTS has been used for GRAINE project for cosmic gamma-ray observation [1], neutrino experiment NINJA [2], cosmic-ray muon radiography project and fast neutron measurement.

\section{Nuclear emulsion readout system and its evolution}

The nuclear emulsion is a three dimensional track detector capable of recording the tracks of charged particles with submicron spatial resolution. The tracks are read by automatic nuclear emulsion readout system which is a combination of microscope, automatic stage and computers. The speed of the readout system has been improving every generation. S-UTS developed in 2006 realized a readout speed of $72 \mathrm{~cm}^{2} / \mathrm{h}$ [3]. The Hyper Track Selector (HTS), which was newly developed in 2015, was designed with the speed of more than 100 times faster than the previous model as shown in Fig. 1. Now, the HTS has operated at the speed of $4700 \mathrm{~cm}^{2} / \mathrm{h}$ at maximum.

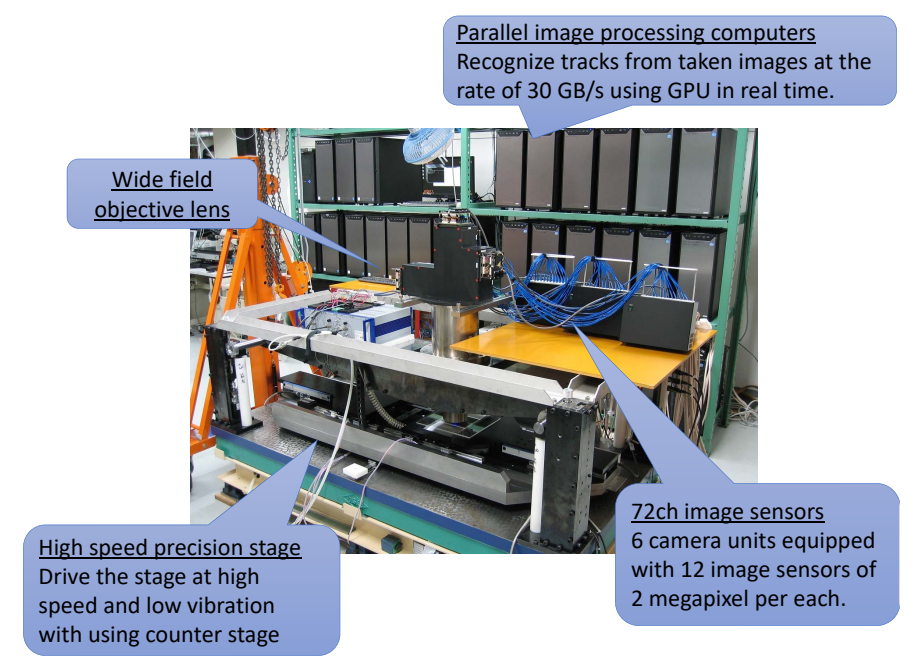

Figure 1: Photo of the HTS and its components.

\section{Technology of HTS}

The largest feature of the HTS is that the field of view is 500 times as large as that of the previous. In order to digitize the images at the same pixel interval as the previous, more than 100 million pixels are required. To realize the high resolution and high frame rate, a field of view is divided into 72 areas and the 72 image sensors are used. The HTS reads tomographic images at a speed of $\sim 30 \mathrm{~GB} / \mathrm{s}$ using 72 high-speed image sensors. The HTS outputs the tracks data from the obtained images in real time with the parallel processing of 36 computers. 


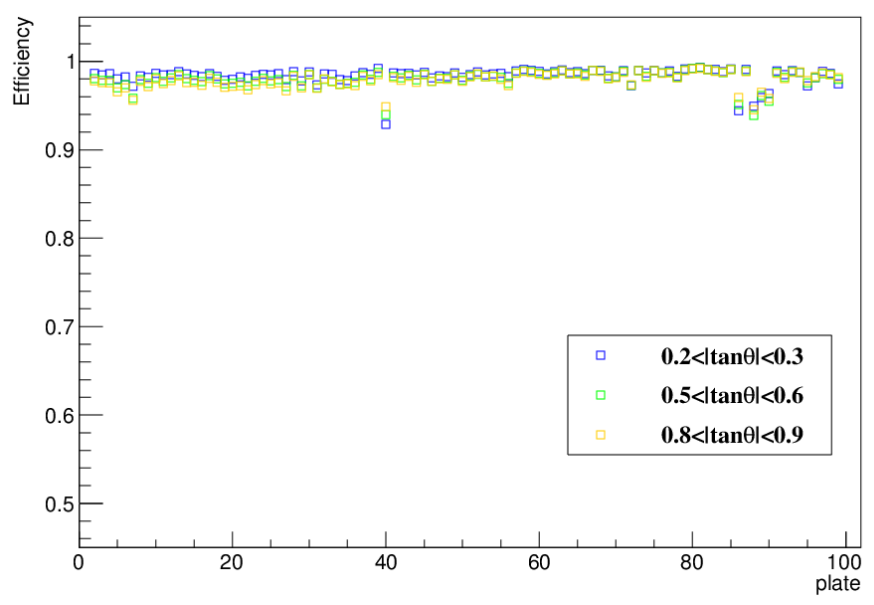

Figure 2: Track finding efficiency of the GRAINE 2015 films.

\section{Calibration of sensor alignment}

Each sensor needs affine parameters for coordinate transformation, because the HTS reads images in parallel with multiple sensors. The parameters sometimes change. Therefore, we have developed a method to calibrate this affine parameters.

All the sensors of HTS have four adjacent sensors. We developed a method to find appropriate positions of all sensors by using positional displacement with using real tracks of two adjacent sensors. The positional accuracy of the track becomes less than $0.3 \mu \mathrm{m}$. As a result, assuming a plastic base of $170 \mu \mathrm{m}$, the angular accuracy of the base track corresponds to less than $2 \mathrm{mrad}$. The details will be written in other papers.

\section{Performance evaluation}

We evaluated base track finding efficiency and angular accuracy by using a nuclear emulsion films used for GRAINE 2015 flight [4]. The track finding efficiency was defined as the proportion of the number of tracks found in a certain film to the number of tracks that could be connected by the next film. As shown in Fig. 2, the detection efficiency of almost all the plates in the angle range of $\theta<45$ degree is $95 \%$ or more. Also, the angular accuracy of the tracks was evaluated. As shown in Fig. 3, the angular accuracy of 3 mrad or less on vertical tracks and less than $8 \mathrm{mrad}$ in the angle range of $\theta<45$ degree. The details will also be written in other papers.

\section{A new approach of HTS for short track recognition}

The HTS usually covers the penetrating tracks through nuclear emulsion films, i.e. high momentum tracks. However, we also want to detect halfway tracks, i.e. tracks started or stopped in emulsion layer, because the recoil nuclei induced by fast neutron, short lifetime particles and low energy tracks are also important.

Now, we have simulated the track finding efficiency for the halfway tracks under the similar condition to HTS. We created tracks of various angles for each length and got the finding efficiency 


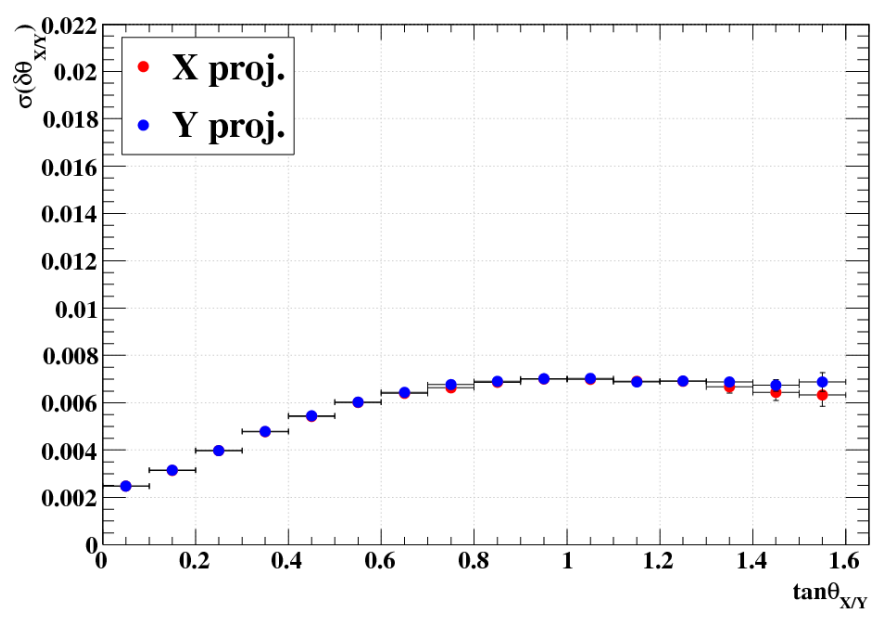

Figure 3: Angle dependence of angle accuracy for base tracks.

by the tracking program with maximum angular space $\tan \theta<2.7$. The results are shown in Fig. 4 . Even though the track length is $20 \mu \mathrm{m}$, we found that the efficiency is more than $40 \%$.

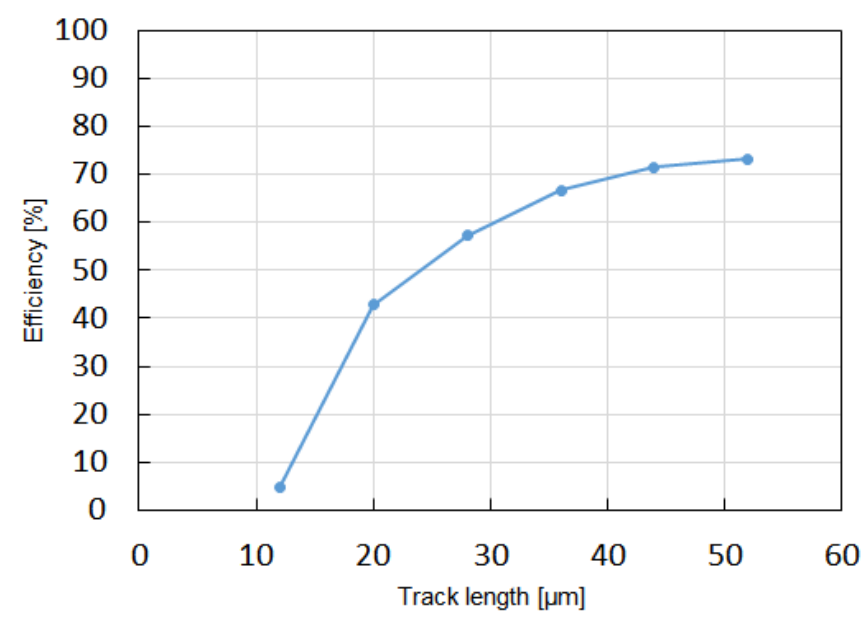

Figure 4: Length dependence of track finding efficiency.

\section{Summary and prospect}

We developed a new automatic nuclear emulsion readout system HTS that realizes $4700 \mathrm{~cm}^{2} / \mathrm{h}$. It is actually used for GRAINE experiments, neutrino experiments NINJA project, fast neutron measurement and so on. In these experiments, it is important to understand the track finding efficiency of short tracks of the order of $10 \mu \mathrm{m}$. We evaluated the detection efficiency for short tracks less than the thickness of emulsion layer. We will investigate the actual track finding efficiency in HTS. 


\section{References}

[1] S. Aoki et al., Balloon-borne gamma-ray telescope with nuclear emulsion: overview and status, arXiv preprint 1202.2529 (2012) [astro-ph/1202 .2529].

[2] S. Fukuda et al., First neutrino event detection with nuclear emulsion at J-PARC neutrino beamline, arXiv preprint 1703.03659 (2017) [hep-ex/1703.03659].

[3] K. Morishima and T. Nakano, Development of a new automatic nuclear emulsion scanning system, S-UTS, with continuous 3D tomographic image read-out, JINST 5 P04011 (2010)

[4] K. Ozaki et al., Development of new-type nuclear emulsion for a balloon-borne emulsion gamma-ray telescope experiment, JINST 10 (12) P12018 (2015) 